Résumés des conférences et travaux

\title{
Histoire de l'Extrême-Orient prémoderne et épigraphie chinoise
}

Pierre Marsone

\section{OpenEdition}

\section{Journals}

Édition électronique

URL : https://journals.openedition.org/ashp/1220

DOI : 10.4000/ashp.1220

ISSN : 1969-6310

\section{Éditeur}

Publications de l'École Pratique des Hautes Études

\section{Édition imprimée}

Date de publication : 1 octobre 2011

Pagination : 272-281

ISSN : 0766-0677

\section{Référence électronique}

Pierre Marsone, "Histoire de l'Extrême-Orient prémoderne et épigraphie chinoise », Annuaire de l'École pratique des hautes études (EPHE), Section des sciences historiques et philologiques [En ligne], 142 | 2011, mis en ligne le 23 septembre 2011, consulté le 06 juillet 2021. URL : http://journals.openedition.org/ ashp/1220 ; DOI : https://doi.org/10.4000/ashp.1220 


\title{
HISTOIRE DE L'EXTRÊME-ORIENT PRÉMODERNE ET ÉPIGRAPHIE CHINOISE
}

\author{
Maître de conférences : M. Pierre MARsone
}

Programme de l'année 2009-2010: Histoire des empires sinisés (Liao, Jin) et de la Chine sous les Mongols : histoire de l'empire des Khitan (907-1125).

\section{Le règne de Taizong (1 ${ }^{\text {re }}$ partie, 926-938)}

Durant les conférences de cette année, nous avons poursuivi la lecture continue et commentée de l'histoire dynastique officielle des Khitan-Liao, le Liaoshi. L'année précédente nous avait amenés à la dernière année de la vie du fondateur de l'empire, Yelü Abaoji (872-926). Les pages qui suivent présentent un matériau de base, la traduction, légèrement résumée, du texte des annales historiques, qui fut réalisée dans son intégralité avec les auditeurs. Les nombreuses annotations et les commentaires auxquels ce texte a donné lieu durant les conférences seront développés ultérieurement dans diverses publications.

Après avoir prophétisé en 924 qu'il lui restait « deux choses à réaliser » avant de « s'en retourner au ciel », Abaoji, qui se considérait comme une incarnation de la divinité, mena à bien ces deux missions qui s'avérèrent être la conquête, à l'ouest, du Sud de la Mongolie jusqu'au Nord du Xinjiang, et, à l'est, du royaume de Bohai. Ce furent deux guerres-éclair couronnées de succès. Bohai, situé entre l'actuelle Corée et le Sungari, continua toujours d'être appelé Dan-gur en khitan, il fut renommé Dongdan en chinois. Sa capitale, Huhancheng, tomba à la première attaque et son nom fut changé en Tianfu, «Bonheur céleste ». Mais dans les préfectures, la résistance s'organisa et dura plusieurs mois. Au $3^{\mathrm{e}}$ mois de 926, Abaoji envoya le juge (yilibi) Kang Moji (?-926) et le vice-président de gauche du département des Affaires d'État, Han Yanhui (882-959), attaquer la préfecture de Changlingfu. Il fit un sacrifice au Ciel et visita dans son palais le «Roi auguste de l'Homme », Bei, son fils aîné, qu'il avait placé à la tête de Dongdan. Mais dans le même temps, les trois préfectures de Anbian, Maoxie et Dingli se rebellèrent, et le plus jeune des frères d'Abaoji, Anduan (?-952), fut envoyé pour les réprimer. Les troupes khitan devaient avoir une supériorité écrasante car elles réduisirent ces trois révoltes en huit jours. Alors qu'Anduan présentait ses prisonniers, les deux meneurs de la révolte d'Anbianfu furent exécutés. Un banquet fut offert aux fonctionnaires de Dongdan et ceux-ci reçurent des récompenses selon leur rang. Deux mois plus tard, la préfecture de Nanhai, capitale du Sud de Bohai, se révolta à leur tour, accompagnée de Dingli qui tenta une nouvelle révolte. Le deuxième fils d'Abaoji, le Grand maréchal Yaogu, les réprima en moins d'un mois. Pendant ce temps, Abaoji visita une dernière fois la capitale Tianfu, puis il se retira avec ses troupes, emmenant le roi vaincu, Tae In-son, et tout son clan. Tae In-son fut transféré à l'ouest de la capitale impériale khitan où une cité fortifiée fut construite pour lui. Le plus sévère châtiment qu'Abaoji lui réserva semble être d'avoir donné, à lui et à son épouse, les noms 
humiliants de Wulugu et Alizhi, les noms des chevaux sur lesquels Abaoji et l'impératrice Chunqin avaient reçu la reddition du couple royal vaincu. Yelü Bei, avec sa cour, raccompagna son père jusqu'aux frontières de Dongdan.

Alors qu'Abaoji faisait halte à Shenzhou, un émissaire des Tang Postérieurs, Yao Kun, vint annoncer le deuil de sa nation. Li Siyuan, le fils adoptif du souverain des Tang, s'était en effet rebellé contre son père, Li Cunxu (Zhuangzong), qui était mort au cours des événements dans des circonstances obscures, officiellement assassiné par le préfet Guo Congqian, ce qui avait permis à Li Siyuan (Mingzong, 926-934) de monter sur le trône. La Nouvelle histoire des Cinq Dynasties a laissé de cette rencontre entre Yao Kun et Abaoji une pièce d'anthologie : un dialogue qui reflète entre les deux hommes une complicité mêlée de cynisme, et qui serait digne d'une bonne comédie. Mais durant ce $7^{\mathrm{e}}$ mois de 926 , Abaoji tomba malade. Le soir, une grosse étoile filante sembla tomber devant sa tente. Au petit matin, on vit au-dessus des faubourgs de Shenzhou un dragon jaune, long d'un $l i$, qui tournoyait dans les airs. Diffusant une clarté aveuglante, il rentra dans le campement. Des vapeurs pourpres et noires obscurcirent le ciel jusqu'au lendemain. Ce jour-là, l'empereur mourut, âgé de 55 ans. Sa prédiction s'était réalisée.

Dès le lendemain du décès, alors que Kang Moji poursuivait l'attaque de Changlingfu, l'impératrice proclama la régence pour assurer provisoirement les affaires militaires et civiles. Elle retourna vers l'ouest en accompagnant le «palais de catalpa » (le cercueil de l'empereur) et fut rejointe par Yaogu puis par Bei. Au 9e mois, la dépouille arriva à la capitale et fut provisoirement déposée dans le faubourg nordouest. L'auguste défunt reçut le nom posthume de Shengtian huangdi (« Empereur monté au ciel ») et le nom de temple Taizu (« Grand Ancêtre »). Au $8^{\mathrm{e}}$ mois de 927, il fut inhumé au mausolée ancestral (Zuling), à $30 \mathrm{~km}$ de l'actuelle ville de Balin zuoqi en Mongolie Intérieure. Pour garder et entretenir le mausolée, on créa la préfecture de Zuzhou («préfecture de l'Ancêtre ») et commanderie de Tiancheng («Ville Céleste »), dirigée par un commissaire impérial.

Le deuxième chapitre de l'Histoire des Liao s'achève sur une louange (zan) qui affirme que les ancêtres des Khitan-Liao descendent de l'empereur mythique Yandi, c'est-à-dire Shennong, et que ce que l'on sait des Khitan commence avec le mythique empereur Qishou. L'épigraphie confirme que, dès les premières décennies de l'empire, les Khitan revendiquaient leur appartenance à la descendance de Qishou et des Xianbei par la lignée de Tanshihuai (r. 156-181), le " grand chef » (da chanyu) qui fédéra les Xianbei face aux Xiongnu et à la Chine. Il est évidemment impossible de prouver que les membres du clan impérial, les Yelü, étaient des descendants de Tanshihuai. Mais cette filiation était certainement la plus prestigieuse, et elle seule convenait à la nouvelle famille régnante. Plus précisément, les Khitan descendent d'une branche des Xianbei, les Xianbei Yuwen, qui donna naissance aux Khitan et aux Kumo Xi. Selon la tradition reprise par les compilateurs de l'Histoire des Liao (Liaoshi), les Yuwen Kumo $\mathrm{Xi}$ et Khitan seraient, comme les souverains Yuwen de la dynastie des Zhou du Nord (557-581), des descendants de Shennong. Mais une tradition plus importante encore, attestée par certains extraits du Liaoshi et surtout par l'épigraphie, rattache les Khitan à l'Empereur Jaune (Huangdi) à travers son vingt-cinquième et dernier fils, Changyi shaozi, qui aurait hérité des terres du Nord. Cette tradition n'est pas isolée puisqu'elle 
trouve un écho dans plusieurs sources historiques chinoises et que les mêmes compilateurs du Liaoshi qui font descendre les Khitan de Shennong reconnaissent que, dans le Huangchao shilu qu'ils purent probablement consulter, Yelü Yan faisait descendre les Khitan de l'Empereur Jaune. Si deux cents ans après la chute de la dynastie, les lettrés des Yuan choisirent de suivre le Livre des Zhou et de rattacher les Khitan à Shennong, ce n'est pas en raison de preuves historiques, mais plus probablement dans l'intention de diminuer le prestige d'un peuple qui tombait dans l'oubli.

Selon la louange, Qishou naquit au mont Du'an, inconnu par ailleurs, et il émigra sur les bords de la rivière Jaune (Huangshui). Ce n'est que lorsque le pouvoir arriva à Yali/Nieli (Niar le Singen), vers 735, que l'on institua des règlements, que l'on créa une administration, que l'on grava les contrats dans le bois et que l'on creusa des étables. Yali, qui laissa étrangement le trône à Zuwu khagan et à la lignée des qaghan Yaonian, engendra Pidie (Yizu) qui combattit les Shiwei jaunes et dont les flèches pouvaient, dit-on, transpercer plusieurs épaisseurs de cotes de maille. Pidie engendra Yundeshi (Xuanzu) qui commença à enseigner au peuple à labourer et à pratiquer l'élevage. Yundeshi engendra Saladi (Dezu), un chef bon et sobre, qui enseigna au peuple à fondre le métal. C'est à son époque qu'on aurait commencé à frapper la monnaie. Le frère cadet de Dezu, Shulan Shilu, soumit au Nord des Ugul et des Shiwei. Au Sud, il pilla les préfectures de Yi et de Ding. C'est à cette époque que commença la construction de villes fortifiées. Saladi-Dezu enseigna au peuple à cultiver le mûrier et le chanvre et à se familiariser avec le tissage. Ayant la volonté d'agrandir son territoire et de multiplier sa population, il céda le pouvoir à Taizu, Abaoji, qui fonda la nation et conquit de vastes territoires depuis la mer de l'Est jusqu'aux sables mouvants de l'Ouest.

\section{Le troisième chapitre $d u$ Liaoshi}

Le règne de l'empereur Taizong (926-947) des Liao est développé sur les chapitres 3 et 4 des annales historiques. Le troisième chapitre commence, comme il se doit, par présenter le nouvel empereur. Son nom personnel khitan est transcrit en chinois Yaogu ou Yaoguzhi. Son nom chinois est Deguang et son surnom Dejin. Il est le deuxième fils d'Abaoji et de l'impératrice Chunqin. Lorsqu'il naquit en 902, on vit une lumière divine extraordinaire, et des chasseurs prirent un cerf blanc et un aigle blanc, ce qui fut interprété comme un signe de bon augure. Quand il eut grandi, il avait l'air grave et sérieux, mais il était bon et décidait de la plupart des affaires de l'armée et de la nation. À l'âge de 20 ans, il fut nommé Grand maréchal de la cavalerie (Tianxia bingma dayuanshuai) et, peu après, il reçut de son père l'ordre d'emmener six armées piller les terres du Sud. En 923, il prit Pingzhou dont il captura le préfet, Zhao Siwen, et le général auxiliaire, Zhang Chong, et il défit les Xi du chef Huxun. Il soumit de nombreuses tribus, pilla les préfectures de Zhenzhou, Dingzhou et Youzhou, et se fit craindre de tous ses voisins. Il suivit son père pour défaire les Ugul, réprimer les Tangut à l'ouest du fleuve, faire tomber les bourgs du Shanxi et prendre l'ancienne ville de Balghasun sur l'Orkhon. Il fut aussi le principal artisan de la conquête de Bohai. 
Contrairement à ce qu'on aurait attendu, ce n'est pas le prince héritier et roi de Dongdan, Yelü Bei, qui succéda à Abaoji, mais son premier frère cadet, Yaogu/ Deguang. Selon la version officielle, au $11^{\mathrm{e}}$ mois de 927 , soit après un an de régence de Chunqin, Bei, à la tête de tous les ministres, présenta à l'impératrice cette demande : « Les mérites et la popularité du fils impérial le Grand maréchal faisant l'unanimité, tant dans le pays qu'à l'étranger, il convient que celui-ci reçoive le pouvoir. » L'impératrice suivit son avis et, le jour-même, Yaogu monta sur le trône. Cette abdication est étrange, et tout porte à croire qu'elle ne fut pas spontanée. L'impératrice Chunqin, qui avait toujours suivi de près les affaires politiques, veilla particulièrement à prendre en main la gestion de la succession. Quelques semaines avant la mort du souverain, elle avait déjà fait assassiner un frère d'Abaoji, Yindishi, envoyé à Dongdan pour assister le nouveau roi. Selon la version officielle, au moment de la mort de son mari, elle voulait, conformément à la coutume khitan, accompagner son mari dans l'au-delà, mais les ministres l'auraient suppliée de rester en vie. Elle aurait alors consenti à ce qu'on ne lui coupe qu'une main qui serait placée avec la dépouille d'Abaoji. Cette version est sans doute éloignée de la réalité. Au moment de la mort d'Abaoji, son autre frère, Diela, et son demi-frère moururent sans que les circonstances de leur mort soient précisées. À la même époque, les officiers chinois Zhang Chong et Lu Guoyong, qui s'étaient ralliés aux Khitan, s'enfuirent et retournèrent chez les Tang Postérieurs. Un parangon de vertu, Yelü Duozhen, fut jeté en prison et y mourut l'année suivante. Son frère cadet, Yelü Tulübu, entra dans la clandestinité mais fut ensuite heureusement rappelé par Taizong. Enfin, trois mois après la mort d'Abaoji, l'histoire officielle mentionne plusieurs exécutions de chefs tribaux et commandants militaires. La réunion de ces informations éparses dans les textes laisse penser qu'à la mort d'Abaoji, Chunqin entreprit une véritable purge pour garder la vie et organiser la succession comme elle l'entendait. Or son projet, en matière de succession, était de faire passer son deuxième fils, Yaogu, peut-être parce qu'elle avait plus confiance dans le caractère guerrier de Yaogu que dans la sensibilité littéraire, artistique et sinophile de Bei.

À l'automne 927, après une visite au temple dédié à son père et la célébration d'un holocauste (rite chaice), Yaogu retourna à la capitale. Dans la salle de la Proclamation de la politique (Xuanzhengdian), les ministres lui présentèrent le titre honorifique d' « Empereur successeur du Saint ». Une grande amnistie fut proclamée et les services compétents proposèrent de changer le nom d'ère mais le nouvel empereur refusa. $\mathrm{Au}$ $12^{\mathrm{e}}$ mois de 926, la mère d'Abaoji, encore vivante, Yanmujin (ca 855-934), fut investie du titre de « Grande impératrice douairière », tandis que Chunqin recevait le titre de « Impératrice douairière qui correspond au Ciel » (Yingtian). Quant à l'épouse de Taizong, elle fut nommée impératrice. L'année se finit paisiblement; le nouvel empereur offrit un sacrifice au Ciel et à la Terre et il envoya des émissaires vers les différents pays. Il inspecta les troupeaux dans le faubourg, les étendards et les tambours, insignes de pouvoir, ainsi que les bureaux et fonctionnaires de tous les services. Il visita le temple de son père et les Jurchen furent les premiers à lui apporter le tribut.

$\mathrm{Au} 1^{\mathrm{er}}$ mois de 928, Taizong passa en revue les soldats de diverses tribus : Ke du Nord (probablement des Xi), Pishi, Yela et Moli. Un chef militaire han rallié aux Khitan depuis 921, Wang Yu, fut nommé commissaire impérial de l'armée de Xingguojun (basée à Longhuazhou) et chef du Secrétariat central. Les annales ajoutent 
que Taizong résida quelque temps au lac Changpo, que l'administrateur de la maison impériale (tiyin) Nieligun lui offrit un loup blanc, puis qu'il alla chasser vers l'est, aux monts Gulishan et au Songshan. Au $3^{\mathrm{e}}$ mois, Wang Du, commissaire impérial de Yiwu chez les Tang, envoya un messager annoncer que Dingzhou voulait se rallier. Taizong depêcha immédiatement le chef xi Tiela pour les secourir.

$\mathrm{Au} 4^{\mathrm{e}}$ mois, l'empereur poursuivit sa route vers l'est et sacrifia au dieu-cerf. Sur le lieu de la chasse, il mobilisa toutes les ressources publiques et privées pour accumuler des plumes, du cuir et du bois, puis il chassa aux monts Sanshan. Il envoya aussi Nieligun et Chala en renfort à Tiela qui, à Dingzhou, venait de repousser une attaque du général Wang Yanqiu. Au $5^{\mathrm{e}}$ mois, il chassa au mont Suolashan et envoya Tulübu réprimer les Ugul. Les Jurchen apportèrent à nouveau le tribut. Le $6^{\mathrm{e}}$ mois, au jour jimao, Taizong célébra une cérémonie pour la pluie (rite seseli). Au $7^{\mathrm{e}}$ mois, Tulübu, vainqueur des Ugul, présenta ses prisonniers. Mais du Sud arrivaient de mauvaises nouvelles : les armées des Tang avaient pris Dingzhou, Tiela était mort et plusieurs dizaines de personnes, dont Nieligun et Chala, avaient été capturées. L'empereur regretta amèrement cet échec douloureux et il indemnisa généreusement les familles des généraux et officiers morts au combat. Puis il fit une offrande dans le temple de son père.

$\mathrm{Au} 8^{\mathrm{e}}$ mois, les Tujue apportèrent le tribut. Taizong demanda qu'on élève à Yikunzhou une stèle commémorative en l'honneur de sa mère, "Impératrice douairière Yingtian qui avait mis au monde le saint $»$. Au $9^{\mathrm{e}}$ mois, Tulübu fit parvenir d'autres prisonniers ugul qui furent distribués comme esclaves aux ministres. Ce mois-là, il alla visiter deux fois son frère aîné dans sa demeure. Le but de ces visites n'est jamais précisé. Dans le même temps, il confortait son pouvoir. Son anniversaire fut nommé « fête du don du Ciel » (Tianshoujie), et celui de l'impératrice douairière, « fête de la Paix éternelle » (Yongningjie). Au $10^{\mathrm{e}}$ mois fut célébrée la première fête de Yongningjie puis l'empereur alla visiter le temple de son père. Un émissaire des Tang apporta une flûte de jade. Ce fut ensuite l'anniversaire de l'empereur qui tint audience dans la salle Wuluandian et reçut les vœux de ses ministres ainsi que ceux des émissaires des différents pays.

$\mathrm{Au} 11^{\mathrm{e}}$ mois, Taizong reçut le tribut des Bigude et rassembla son armée pour aller punir les Tang. Mais les cadeaux des Tang se multipliant au $12^{\mathrm{e}}$ mois, les ministres lui conseillèrent de temporiser et il se rangea à cet avis. Ce mois-là, il ordonna de déporter une partie de la population de Dongdan pour peupler Dongping, l'actuel Liaoyang, qui fut renommé « capitale du Sud » (Nanjing), ce qui provoqua un exode de population à Silla et chez les Jurchen. En effet, l’ordre précisant que « les miséreux qui ne pouvaient se déplacer seront autorisés à être nourris par les riches de l'élite et à leur appartenir », on en déduit que la déportation imposée devait être financée par les déportés eux-mêmes.

Au $1^{\text {er }}$ mois de 929, après le banquet du nouvel an, Taizong alla à Guaguo sur la rivière Jaune et il inspecta les foyers du clan Yaonian le mois suivant. Au $3^{\mathrm{e}}$ mois, il sacrifia à tous les esprits. Au $4^{\mathrm{e}}$ mois, revenu de Guaguo, il visita le temple de son père, puis le campement de son père, et il alla ensuite à la commanderie de Tianchengjun visiter le mausolée. Ce même mois, Bei fut reçu en audience et Taizong révisa des procès. Au $6^{\mathrm{e}}$ mois, Tulübu amena de nouveaux prisonniers ugul et les militaires furent 
récompensés, ce qui montre que la soumission des Ugul, malgré des victoires régulières, fut laborieuse. L'empereur partit ensuite vers l'ouest pour chasser avec mille cavaliers d'élite dans les montagnes alentour, et il s'arrêta à Liangxing. Le mois suivant, il visita le marché, amnistia exceptionnellement des prisonniers, fit une offrande à son père et rentra. Au $8^{\mathrm{e}}$ mois, il visita deux fois le temple de son père et alla voir Bei entre ces deux visites. Des problèmes familiaux semblaient le tracasser.

Au $9^{\mathrm{e}}$ mois, Taizong partit pour Nanjing (Liaoyang). En passant au mont sacré Muyeshan, il fit une offrande et célébra la cérémonie de la Renaissance (rite zaishengli) que doit passer un souverain khitan. Au $10^{\mathrm{e}}$ mois, à Nanjing, il rendit visite à Bei qui, quoique toujours officiellement roi de Dongdan, devait partager son temps hors de son royaume, entre la capitale principale et la capitale du Sud des Khitan. Taizong offrit un banquet à ses ministres, passa des troupes en revue et envoya son frère cadet, Lihu, en campagne dans le nord du Shanxi. Au $11^{\mathrm{e}}$ mois, il annonça au Ciel et à la Terre le début de la campagne, donna dans le faubourg ouest un banquet pour le départ de Lihu et ordonna au tiyin d'annoncer le départ des troupes dans le temple et dans le campement d'Abaoji. Puis il alla pêcher à Sanchakou. Au $12^{\mathrm{e}}$ mois, il reçut le tribut des Jurchen et rentra à sa capitale.

$\mathrm{Au} 1^{\mathrm{er}}$ mois de 930, Taizong, réjoui que Lihu eût déjà pris Huanzhou (au Shanxi), rendit visite à l'impératrice douairière. Le mois suivant, il ordonna la fortification de Nanjing. Lihu lui rendit visite et Taizong le récompensa en lui donnant des captifs de Bohai. Puis Taizong retourna avec Bei visiter leur mère, l'impératrice douairière, qui demanda à ses fils de lui faire des démonstrations de calligraphie. Nous ne savons pas si les caractères calligraphiés étaient khitan ou chinois. Au $3^{\mathrm{e}}$ mois, l'empereur visita à nouveau sa mère puis son oncle, Anduan. Il décerna à Lihu les titres de « Cadet impérial à la longue vie et florissant » et de « Grand maréchal de toute l'armée sous le ciel ». Puis il quitta la capitale du Sud.

$\mathrm{Au} 4^{\mathrm{e}}$ mois, Taizong envoya Bei visiter le temple de leur père au mausolée d'Abaoji, après quoi Bei rentra dans son pays. Durant les mois suivants, Taizong visita au moins une fois par mois un temple de son père. Au $6^{\mathrm{e}}$ mois, on signale un tribut des Diliede, et au $7^{\mathrm{e}}$ mois un tribut des Ugul. Au $8^{\mathrm{e}}$ mois, un lieu où l'Empereur à la Grande sainteté et son l'impératrice avaient vécu fut appelé « Palais du Soleil et de la Lune » et une Stèle $d u$ Soleil et de la Lune fut érigée. L'empereur se rendit ensuite à une « Terrasse à neuf étages » (Jiucengtai) inconnue par ailleurs. Durant son séjour, Taizong tenta apparemment de rassurer son frère Bei en envoyant le commandant Puning le réconforter et en créant pour lui une garde spéciale. Puis il rentra, visita le temple de son père et dépêcha un émissaire apporter à Bei des offrandes provenant du sacrifice. Bei offrit en remerciement une flûte de jade. À ce moment, Taizong fit ériger sur le Lieu des assemblées du Roi d'or (Ruyuwang jihuiwo) une stèle vantant les « saints mérites » de son père.

Toutes les visites de l'empereur à sa mère et dans le temple de son père furent probablement motivées, en partie du moins, par un conflit latent. Ayant été contraint de laisser le pouvoir à son frère, Bei constituait une menace pour Taizong. C'est pour cette raison que Taizong ne laissait plus Bei habiter dans son royaume de Dongdan et qu'il lui attribua une garde chargée de le surveiller au moins autant que de le protéger. Sentant probablement sa vie menacée, Bei prétexta une partie de pêche et s'enfuit chez les Tang par la mer, avec une concubine, au $11^{\mathrm{e}}$ mois. 
$\mathrm{Au} 1^{\text {er }}$ mois de 931, le général de la frontière sud-ouest annonça qu'il avait rallié des Kirghizes, et les Diliede apportèrent le tribut. L'empereur retourna à la capitale du Sud où l'épouse principale de Bei, qui était restée en territoire khitan, vint le saluer. Au $4^{\mathrm{e}}$ mois, un émissaire des Tang apporta des cadeaux et le gouvernement de Dongdan (Zhongtaisheng) fut transféré à la capitale du Sud. L'empereur revint à la capitale principale en s'arrêtant au mont Muyeshan où il fit une offrande. Au $6^{\mathrm{e}}$ mois, il alla à Liangxing et les Ugul apportèrent le tribut, suivis par les Jurchen le mois suivant. $\mathrm{Au} 8^{\mathrm{e}}$ mois, le premier fils de Taizong, Shulü, naquit, et l'empereur annonça solennellement la nouvelle dans le temple de son père. Les Bigude apportèrent le tribut. $\mathrm{Au}$ $9^{\mathrm{e}}$ mois, l'empereur ordonna de restaurer les murailles de la capitale. Les Tieli et les Tang apportèrent aussi un tribut et des cadeaux au cours des mois suivants.

Au début de 932, Yela Dide effectua auprès du royaume de Wu Yue une mission qui permit de renouer les relations entre les deux États. $\mathrm{Au} 4^{\mathrm{e}}$ mois, les Tang envoyèrent des présents et apportèrent une lettre de Bei en exil. Au $5^{\mathrm{e}}$ mois, Taizong visita le mausolée de son père et au $6^{\mathrm{e}}$ mois il ordonna d'ériger la Stèle de la fondation de la Nation par Taizu. Les Ugul et les Diliede apportèrent le tribut. Les annales insistent ensuite sur l'attention de l'empereur pour sa population : au $7^{\mathrm{e}}$ mois, des cadeaux furent distribués à tous les fonctionnaires et l'empereur offrit des étoffes aux personnes âgées. Le mois suivant, alors que Taizong chassait l'oie sauvage au lac Yanliuhu, une tempête renversa des bateaux. Plus de soixante personnes périrent noyées et l'empereur ordonna d'indemniser leurs familles.

$\mathrm{Au} 1^{\mathrm{er}}$ mois de 933, Lihu partit en campagne contre les Tangut et revint victorieux deux mois plus tard. Les Tangut apportèrent le tribut et les Ouighours Arslan (Ouighours de Turfan) commencèrent aussi à apporter le tribut. Au $10^{\mathrm{e}}$ mois, les Zubu, les Tang et les « Ugul Tulumo » apportèrent le tribut. Au $11^{\mathrm{e}}$ mois, la grande impératrice douairière mourut, tout comme le souverain des Tang, Li Siyuan, auquel son fils, Li Conghou, succéda. Au $12^{\mathrm{e}}$ mois, les Tangut apportèrent le tribut.

Au premier mois de 934, alors que l'empereur pêchait sur le Tuhe, les Tangut apportèrent un tribut de chameaux et de cerfs tandis que la capitale du Sud offrait un cerf des marais. Au $2^{\mathrm{e}}$ mois, la grande impératrice douairière fut inhumée dans le mausolée de son époux, le "mausolée de la Vertu » (Deling), du nom de son époux qui avait reçu le titre posthume de «Ancêtre vertueux » (Dezu). Elle reçut le titre posthume d'impératrice Xuanjian et une stèle fut érigée. Au 4è mois, chez les Tang, Li Congke tua son frère et prit le pouvoir. Bei envoya une lettre pour suggérer aux Khitan d'intervenir. Au $8^{\mathrm{e}}$ mois, Taizong prit lui-même la tête des armées en direction du sud. Les annales notent que Yila Jieli attrapa à la main une oie sauvage en plein vol et que l'empereur en fut tellement impressionné qu'il fit une offrande au Ciel et à la Terre. Les Khitan avançaient dans le nord du Shanxi. Au $11^{\mathrm{e}}$ mois ils prenaient Yangcheng. $\mathrm{Au} 12^{\mathrm{e}}$ mois, l'impératrice donna à Taizong un deuxième fils, Abo Sageli, mais elle mourut le mois suivant, probablement des suites de l'accouchement.

$\mathrm{Au} 4^{\mathrm{e}}$ mois de 935, l'été, le chef tuyuhun Tuiyude se rallia avec ses troupes. Le clan du père de l'impératrice douairière et celui du premier mari de sa mère obtinrent le titre d' « oncles de la nation » et furent placés sous la direction du deuxième frère aîné de Chunqin, Xiao Shilu, appelé ici le «shangfu Xiao Miansi ». Au $5^{\mathrm{e}}$ mois, l'impératrice fut inhumée au mausolée Fengling. L'empereur lui-même rédigea un texte dans lequel 
il lui donnait le titre de «Impératrice qui a fait briller la vertu » (Zhangde huanghou). $\mathrm{Au} 11^{\mathrm{e}}$ mois, l'empereur visita un temple bouddhique, le Hongfusi, et nourrit les moines pour le repos de l'âme de l'impératrice. À cette occasion, il vit une image de Guanyin qui avait été offerte par ses parents et son frère. Se tournant vers ses assistants il dit : "Autrefois, nous sommes venus nous recueillir ici avec mon père, ma mère et mon frère. Cela est encore si proche, et me voilà de retour ici, mais seul ! » Il éclata en sanglots et écrivit alors lui-même un poème sur le mur pour exprimer son sentiment. Les annales ajoutent que ceux qui virent cette scène en furent profondément émus.

$\mathrm{Au} 1^{\mathrm{er}}$ mois de 936, Taizong pêcha de nouveau sur le Tuhe, puis il alla au Huanghe. $\mathrm{Au} 3^{\mathrm{e}}$ mois, des Jurchen apportèrent le tribut. Au $4^{\mathrm{e}}$ mois, l'empereur visita deux fois le mausolée de son père. Il reçut à nouveau un tribut de Jurchen et offrit des vêtements aux émissaires ouighours. Au $6^{\mathrm{e}}$ mois, les Bigude et des Tuyuhun apportèrent le tribut. $\mathrm{Au} 7^{\mathrm{e}}$ mois, des Ugul apportèrent le tribut et la princesse Pugening, à la tête des « Ugul des trois rivières », vint en audience. Shi Jingtang fit demander de l'aide par l'intermédiaire du commissaire à la répression du Sud-Ouest, Lubugu. L'empereur dit à l'impératrice douairière : «Li Congke a tué son souverain et s'est proclamé empereur. Il faut appliquer la répression céleste ». Au même moment, Zhao Dejun envoya un émissaire et le Hedong dépêcha Sang Weihan pour appeler au secours. Alors l'empereur décida de lever des troupes. Au $8^{\mathrm{e}}$ mois, Xiao Xiali déclara la guerre à l'armée du Hedong et Taizong prit lui-même la tête de l'armée pour aller aider Shi Jingtang. Au $9^{\mathrm{e}}$ mois, la chute d'une espèce de cormoran fut interprétée comme un signe faste. L'empereur entra par la passe de Yanmen, célébra un sacrifice au Ciel et à la Terre, à Xinzhou, et arriva à Taiyuan. Il déploya ses troupes et défit les généraux des Tang Zhang Jingda et Yang Guangyuan venus en renfort.

Quand Shi Jingtang arriva avec ses fonctionnaires, l'empereur khitan le reçut chaleureusement et le nomma roi de Jin. Il convoqua Shi Jingtang au campement, le fit asseoir et il lui dit calmement : « J'ai parcouru 3000 li avec mes troupes, et dès la première bataille je suis vainqueur. Cela doit bien être la volonté du Ciel. Tu as l'autorité et la carrure pour recevoir cette terre du Sud et être de génération en génération mon vassal. » Il ordonna alors d'aménager une aire rituelle à Jinyang (ancien nom de Taiyuan) pour l'investir, au $11^{\mathrm{e}}$ mois, du titre de « empereur des Grands Jin ». De son côté, le général Zhang Jingda, encerclé depuis plus de 80 jours, isolé de toutes parts et sans réserves, en était réduit à laver le crottin des chevaux et à nourrir ces derniers de copeaux de bois. Les chevaux étaient affamés au point de se manger mutuellement les crinières et les queues, et quand ils mourraient on les mangeait. Ses compagnons d'infortune pressèrent Zhang Jingda de se rendre mais, devant son refus obstiné, ils le tuèrent et se rendirent. Quand Taizong apprit que Jingda était resté inflexible jusqu'à la mort, il dit à ses assistants : «C'est ainsi que tout ministre doit se comporter » et il le fit enterrer selon les rites. Taizong offrit un sacrifice au Ciel et à la Terre pour annoncer son succès. Zhao Dejun, qui tentait de s'enfuir avec les renforts, fut capturé. Alors que l'empereur des Jin allait s'en retourner, Taizong lui offrit un festin. Quand ils eurent bien bu, Taizong lui prit la main et déclara qu'ils seraient père et fils. Il lui offrit une peau de zibeline blanche, 20 chevaux de ses écuries et 1200 chevaux de guerre, et il ordonna à Dilibi de l'escorter avec 5000 cavaliers jusqu'à Luoyang. Au moment du départ, il lui dit : «Je reste ici et dès que le désordre sera apaisé je m'en retournerai ». 
Lorsque l'empereur des Jin arriva à Heyang, Li Congke était acculé. Il demanda à Yelü Bei de mourir avec lui, mais comme celui-ci refusait, il envoya quelqu'un l'assassiner et toute sa famille s'immola par le feu. On rassembla les dépouilles de ses soldats morts dans les combats et on les inhuma dans un jingguan (grande tombe collective) sur les bords de la rivière Fen. Sang Weihan reçut l'ordre d'écrire un texte pour noter leurs mérites.

$\mathrm{Au} 12^{\mathrm{e}}$ mois, Taizong envoya son assistant, Talu, pour réconforter l'empereur des Jin vraisemblablement inquiet, et il distribua le butin de Jin'an aux généraux et aux officiers, puis il envoya un messager rendre compte des événements à l'impératrice douairière et lui annoncer le retour de toutes les armées. Parti de Taiyuan, il fit halte au Xihe où il passa en revue des troupes de Zhao Dejun et de son fils qui s'étaient rendues. Au $1^{\text {er }}$ mois de l'année suivante (937), il confia à Cui Tingxun la répression de la rébellion de Datong. Après avoir pratiqué un exorcisme de criblage de flèches d'un démon (she guijian) au nord de Yunzhou, il fit un sacrifice au Ciel et à la Terre et il ordonna à son oncle Anduan de raccompagner les Xi occidentaux sur leur terre. (Les Xi s'étaient progressivement rendus aux Khitan à la fin du IX ${ }^{\mathrm{e}} \mathrm{s}$., mais dans les années 907-911 un de leurs chefs, Quzhu, excédé par la dureté des Khitan, s'était réfugié avec sa tribu dans les montagnes du nord de Guizhou, actuellement Huailai, au Hebei, où fut créée la préfecture de Kehanzhou. Les Xi se distinguèrent à partir de ce moment en Xi occidentaux et Xi orientaux.) Le fils aîné de l'empereur, Shulü, accueillit son père à la rivière Luanhe (actuel Shandianhe) et Taizong annonça ses mérites dans le campement de son père. Il fut ensuite reçu par l'impératrice douairière et apporta des jades précieux pour lui souhaiter la longévité.

Au $6^{\mathrm{e}}$ mois de 937, les Jin envoyèrent des émissaires, dont le ministre des Finances, Nie Yanzuo, pour demander de pouvoir offrir un nom honorifique aux empereurs et de se voir rétrocéder des territoires au nord de Yanmen ainsi que les régions de You et de Ji tout en conservant le tribut de 300000 pièces de soie, ce qui leur fut refusé. $\mathrm{Au} 7^{\mathrm{e}}$ mois, Taizong ordonna aux tribus de former des escouades militaires et il alla à Huaizhou où il visita le mausolée de Fengling où était inhumée son épouse. Au $8^{\mathrm{e}}$ mois, Jin envoya à nouveau des émissaires pour demander un titre honorifique, ce qui leur fut encore refusé. Puis un autre émissaire vint annoncer la reddition de la capitale, Bian.

$\mathrm{Au} 10^{\mathrm{e}}$ mois, les Jin, les Ouighours et le pays de Dunhuang envoyèrent des émissaires féliciter l'impératrice douairière à l'occasion de sa fête. Les émissaires ouighours Hulizhi et Alabao furent convoqués en audience pour parler de leurs coutumes, puis ils s'en retournèrent, accompagnés des émissaires khitan Puligu et Humoli le Pishi. Le mois suivant, Taizong fit appeler chez les Jin un médecin qui arriva le mois suivant, après que l'empereur eut fait une nouvelle offrande sur le mont Muyeshan.

\section{Le quatrième chapitre du Liaoshi}

La $1^{\text {re }}$ année de l'ère Huitong (938), lors des vœux des ambassadeurs à la cour, un envoyé des Jin annonça que le grand lettré He Ning (898-955) avait été chargé de rédiger une Stèle des mérites divins de l'action du Saint en l'honneur d'Abaoji. Au $2^{\mathrm{e}}$ mois, des Shiwei apportèrent un daim blanc et l'empereur chassa au mont Songshan. 
Il alla à l'est du Liao et délégua l'administrateur de la maison impériale pour célébrer une offrande à la mémoire de son frère aîné dans le campement de Bei. Il ordonna aussi d'augmenter le nombre des foyers fournis aux émissaires des Jin sur leur passage. Au $4^{\mathrm{e}}$ mois, Taizong arriva à la capitale du Sud et se baigna dans les sources thermales. Rentré au campement, il visita l'impératrice douairière. Les Jurchen apportèrent un tribut d'arcs et de flèches et le grand général du Sud-Ouest, Yelü Lubugu, annonça une victoire sur les Tangut. Le mois suivant, les Jin obtinrent enfin de pouvoir offrir des noms honorifiques. En présence d'un émissaire khitan spécialement venu pour la circonstance, ils donnèrent à leur souverain le titre de « Empereur martial et héroïque qui a compris la justice » (Yingwu mingyi huangdi).

$\mathrm{Au} 6^{\mathrm{e}}$ mois, Taizong ordonna qu'on construise une « Salle du Soleil, de la Lune et des Quatre saisons ", et que les hauts-faits des empereurs d'autrefois fussent peints sur ses bâtiments latéraux. Au $9^{\mathrm{e}}$ mois, les gardes des frontières annoncèrent que les Jin envoyaient le suppléant au ministre des travaux publics, Feng Dao, et le cavalier de l'escorte impériale, Wei Xun, pour apporter un titre honorifique à l'impératrice douairière, ainsi que le vice-président de gauche du département des Affaires d'État, $\mathrm{Liu} \mathrm{Xu}$, avec le conseiller censeur de droite de l'empereur, Lu Zhong, pour apporter un titre à l'empereur khitan. Au $11^{\mathrm{e}}$ mois, les premiers ministres du Sud et du Nord et les yilijin reçurent l'ordre d'aller là où logeait la délégation de l'émissaire des Jin pour leur servir un banquet. Au jour bingwu, l'empereur, dans la salle Kaihuangdian, reçut les émissaires des Jin. Dans cette même salle, au jour renzi, Feng Dao et Wei Xun présentèrent son titre honorifique à l'impératrice douairière. On célébra la cérémonie de la Renaissance (rite chaice) puis, au jour bingyin, Liu Xu et Lu Zhong offrirent son titre à l'empereur dans la salle Xuanzhengdian. Une grande amnistie fut prononcée et l'ère Huitong décrétée.

Ce mois-là, les Jin envoyèrent encore Zhao Ying porteur d'un mémoire de félicitations qui comportait un plan des seize préfectures que les Jin offraient aux Khitan : You, Ji, Ying, Mo, Zhuo, Tan, Shun, Gui, Ru, Xin, Wu, Yun, Ying, Shuo, Huan, Yu. La capitale, Huangdu, fut renommée Shangjing, préfecture de Linhuang. Youzhou fut élevée au rang de capitale du Sud, Xinzhou renommée Fengshengzhou et Wuzhou renommée Guihuazhou. Cette réforme s'accompagna d'une augmentation des titres de fonctionnaires : les adjoints aux magistrats de district (zhubu) devinrent sous-préfets (ling), les sous-préfets (ling) devinrent préfets, et les préfets devinrent commissaires impériaux. Taizong créa au même moment des fonctions administratives nouvelles et modifia de nombreux titres, restructurant en profondeur l'administration. L'empire khitan inclut désormais une administration plus complexe, un assez grand territoire chinois et un empire chinois du Nord était devenu son vassal. Une nouvelle page s'ouvre dans son histoire. 